\title{
Un gène de résistance aux agents anticancéreux gouverne la synthèse d'une protéine de transport
}

La chimiothérapie anticancéreuse se heurte souvent au développement d'une résistance dont le caractère le plus frappant est qu'elle devient rapidement polyvalente et s'exerce à l'encontre de médicaments qui n'ont pas de relation chimique entre eux. Ce comportement est aussi celui de nombreuses lignées cellulaires devenues résistantes. Ce phénomène apparaît lié à un gène ou à un petit nombre de gènes appelés Mdr (multidrug resistance). Les travaux les plus passionnants se sont centrés sur l'étude d'une glycoprotéine de membrane de 170-180 kilodaltons, la gp170 qui semble codée par un gène $\mathrm{Mdr}$ et dont les caractères viennent d'être précisés dans une série de publications.

D'abord, le taux de la gp170 est fortement augmenté dans les lignées résistantes [1] et la résistance diminue sous l'effet d'un anticorps monoclonal dirigé contre la protéine [2]. Ensuite, un ADN complémentaire correspondant à un gène $\mathrm{Mdr}$ a été préparé ; après amplification, sa transfection confere à une cellule sensible les propriétés de résistance. Il s'agit d'un effet purement quantitatif : la surexpression d'un gène $M d r$ normal est suffisante, puisque l'ADN utilisé provient d'une cellule sensible et n'a donc probablement pas subi de mutation [3]. Enfin, l'ADNc d'un gène $\mathrm{Mdr}$ a non seulement été isolé mais entièrement séquencé par deux groupes de chercheurs au Canada et aux USA, chez la séquence déduite, longue d'environ 1280 acides aminés, présente de nombreuses propriétés intéressantes : a) on peut diviser la séquence en deux moitiés qui offrent une très grande ressemblance entre elles. L'interprétation évidente est celle d'une duplication interne d'un gène ancestral ; b) chacune des moitiés comporte une portion hydrophobe suivie d'une portion hydrophile. L'analyse des régions hydrophobes, comparée à celle de protéines de membrane connues, suggère qu'il s'agit d'une protéine membranaire, comportant un certain nombre de boucles transmembranaires. L'ensemble de la séquence porte 8 sites possibles de glycosylation, justifiant une taille apparente de $170 \mathrm{kD}$ alors que le gène $\mathrm{Mdr}$ cloné code pour une protéine de $140 \mathrm{kD}$; c) la séquence présente dans chacune de ses deux parties hydrophiles un site possible de liaison pour un nucléotide ; il existe une séquence analogue dans plusieurs ATPases, ce qui suggère une fixation vraisemblable de l'ATP.

L'ensemble de la structure putative est schématisée dans la figure 1. Elle permet de tenter d'interpréter le mode de fonctionnement de la gp170. L'absorption de médicaments par la cellule se fait par diffusion passive ; leur expulsion au contraire implique un processus actif de pompage contre un gradient de concentration. La structure de la gp170 est compatible avec un rôle dans cette expulsion. Son augmentation dans la cellule résistante expliquerait que la concentration des drogues soit inférieure à celle des cellules sensibles ; l'énergie nécessaire serait fournie par l'hydrolyse de l'ATP.

On a voulu appuyer cette interprétation par la recherche de ressemblances avec d'autres protéines dont la séquence était tirée de banques de données. La surprise est venue de la découverte de fortes homologies avec plusieurs protéines de transport de bactéries Gram négatif. En comparant de nombreux systèmes de transport spécialisés, on a constaté que ce sont les protéines liant l'ATP qui ont la plus forte homologie entre elles, comme avec les portions hydrophiles de la gp170. Par exemple l'homologie avec la protéine hlyB du colibacille (qui sert au transfert des hémolysines) atteint $60 \%$, ressemblance extraordinaire pour des organismes qui ont divergé depuis si longtemps [6].

Il existe cependant des différences : dans les bactéries, ce système de "perméases " forme un complexe dont chaque élément apporte sa spécificité pour le transfert d'un substrat donné ou de quelques substrats. On s'est donc efforcé d'imaginer un modèle reliant les perméases bactériennes et la gp170 [7]. Cette dernière aurait incorporé en une seule molécule les traits de l'ensemble du complexe membranaire bactérien. Il est toutefois probable que d'autres protéines, spécifiques de certaines drogues, les captent pour les livrer ensuite à la gp170. 


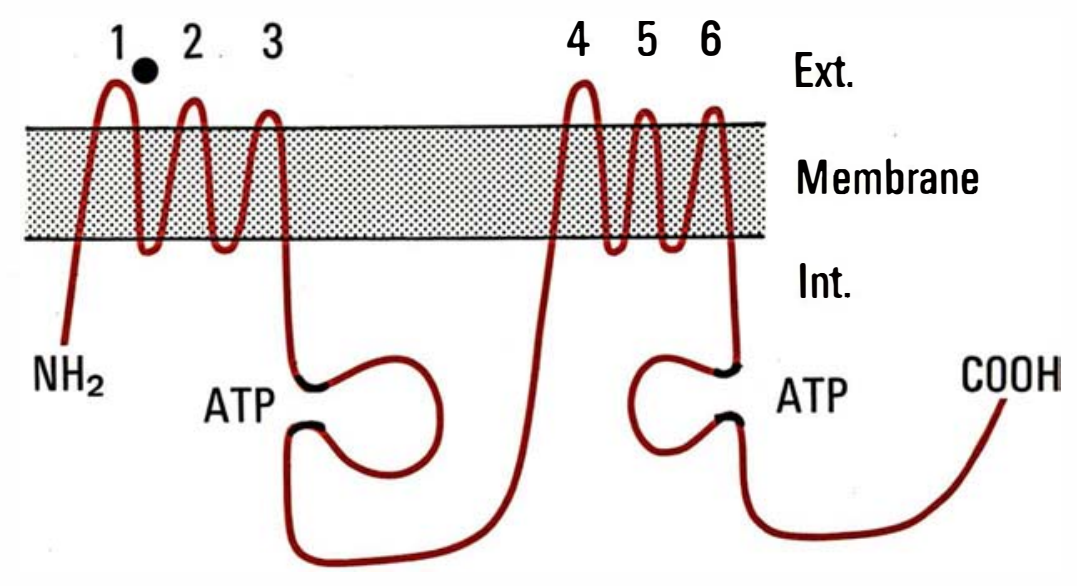

Figure 1. Modèlo schématique do la gp170. (D'après [4] et [5]). EXT : zone extracellulaire ; INT : zone intracellulaire ; 0 : région riche en sites de glycosylation. Les sites de fixation de l'ATP sont également montrés.

Une hypothèse intéressante a été émise par Ames [7]. Le gène $\mathrm{Mdr}$ s'exprime dans les tissus normaux, notamment dans la surrénale. On ignore le rôle physiologique de son produit, la gp170. Il se pourrait que son intervention dans le transport des drogues ne soit qu'un "détournement " de ce rôle normal. L'élucidation de sa fonction pourrait conduire à modeler des agents thérapeutiques qui ne soient pas reconnus par ce barrage.

Enfin, il ne faudrait pas croire que, malgré l'intérêt puissant du système Mdr-gp 170, il ne puisse y avoir d'autres mécanismes qui interviennent dans la résistance aux médicaments. C'est ainsi qu'un travail récent fait état d'une multiplication par 45 de l'activité de la glutathion-transférase (avec apparition d'un isozyme jusqu'alors connu seulement dans le placenta) dans des cellules de cancer du sein humain, résistantes à l'adriamycine [8].

J.-C. D.
1. Riordan JR, Deuchars K, Kartner N, Alon N, Trent J, Ling V. Amplification of Pglycoprotein in multidrug mammalian resistant cell lines. Nature $1985 ; 316: 817-9$. the 170 to $180 \mathrm{kD}$ glycoprotein specific to drug-resistant tumor cells as revealed by monoclonal antibodies. $J$ Biol Chem 1986; 83 : 7785-9.

3. Gros P, Ben Neriah Y, Croop JM, Housman DE. Isolation and expression of a complementary DNA that confers multidrug resistance. Nature 1986 ; 323 : 728-31.

4. Gros P, Croop J, Housman D. Mammalian multidrug resistance gene : complete DNA sequence indicates strong homology to bacterial transport proteins. Cell $1986 ; 47: 371-80$. 5. Chen CJ, Chin JE, Ueda K, et al. Internal duplication and homology with bacterial transport proteins in the mdrI (P-glycoprotein) gene from multidrug-resistant human cells. Cell $1986 ; 47: 381-9$.

6. Gerlach JH, Endicott JA, Juranka PF, et al. Homology between P-glycoprotein and a bacterial haemolysin transport protein suggests a model for multidrug resistance. Nalure 1986 ; 324 : 485-9.

7. Ames GFL. The basis of multidrug resistance in mammalian cells : homology with bacterial transport. Cell $1986 ; 47: 323-4$.

8. Batist G, Tulpule A, Sinha BK, et al. Overexpression of a novel anionic glutathione cransferase in multidrug-resistant human breast cancer cells. J Biol Chem $1986 ; 261$ : 15544-9.
2. Hamada $H$, Tsuruo T. Functional role for

\section{BRÈVES}

- Le parasite Trypanosoma cruzi, agent de la maladie de Chagas, adhère aux cellules qu'il va infecter par l'intermédiaire de la fibronectine. Cette adhésion est inhibée par des peptides synthétiques contenant la séquence ArgGly-Asp-Ser (RGDS) qui caractérise les sites d'attachement des cellules de mammifères et de virus à la fibronectine $\left(\mathrm{m} / \mathrm{s} n^{\circ} 2\right.$, vol. 2, p. 337 et 589). Des anticorps anti RGDS inhibent également l'adhésion du parasite et la vaccination de la souris par un antigène contenant ce motif peptidique confere une résistance relative à l'infestation parasitaire.

Ouaissi MA, et al. Science 1986 234 : 603-7.

Les mutations ponctuelles ne sont pas régulièrement réparties dans un gène. Les dinucléotides $\mathrm{CpG}$ sont des "points chauds" (hot spot) de mutations car les résidus $\mathrm{C}$ sont, chez les mammiferes, massivement méthylés et, les 5-méthylcytosines étant instables, subissent fréquemment une désamination, entraînant une transition $\mathrm{C} \rightarrow \mathrm{T}$. $\mathrm{Ce}$ phénomène est bien illustré par l'étude des gènes du facteur VIII chez 83 hémophiles A. Deux mutations particulières pouvant résulter d'une telle transition $\mathrm{C} \rightarrow \mathrm{T}$ ont été ainsi découvertes dans des familles d'origines différentes signant bien l'existence de " points chauds " dans le gène qui contient plus de 20000 bases codantes. Dans les deux cas, ces mutations entraînent l'apparition d'un codon stop et sont apparues chez le grand-père maternel.

Youssoufian $\mathrm{H}$, et al. Nature $1986 ; 324$ : 380-2. 